\title{
Testing the two-stream instability in a pulsar magnetosphere
}

\author{
Estelle Asseo \\ Centre de Physique Theorique CNRS Ecole Polytechnique 91128 \\ Palaiseau France
}

\begin{abstract}
Conditions for the development of a two-stream instability in a pulsar magnetosphere are deduced from specific dispersion relations for plane wave perturbations, that depend on the distribution functions of the involved particles. Three different approaches are investigated.

Firstly, using relativistic one-dimensional Juttner Synge distribution functions appropriate to describe pulsar pair plasmas flows, we analytically derive the dispersion relation anew, precisely determining the dependence of its coefficients on the temperature, fluid velocity and associated Lorentz factor. We obtain modified frequencies for quasi-longitudinal waves and specific conditions for a two-stream instability to develop.

Secondly, the importance of two-stream instabilities is tested numerically on two different timescales, that concern stationary and nonstationary properties of pair plasma flows. The linear analysis involves Gaussian distribution functions of the momentum, factorized with functions that depend on the localisation of the different groups of particles, and shows results that agree with observed luminosities.

Finally as derived from fluid equations, the nonlinear evolution of such an instability process allows to associate the high level of radio radiation observed from pulsars with the existence of a lattice of radiating 'Langmuir' soliton-like structures in a pulsar emission region. Actually, pair plasma particles follow the bundle of diverging magnetic field lines in the open magnetosphere and 'Langmuir' soliton-like solutions, modified by magnetic field and density gradients, imply additional radiation.
\end{abstract}

\section{Two-stream instability in pulsar magnetospheres}

The possibility for a two-stream instability to be responsible for pulsar radio radiation concern three typical interactions able to develop in a pulsar magnetosphere : 1./ the interaction between the primary beam and the secondary pulsar pair plasma (Ruderman \& Sutherland 1975), 2./ the interaction between electrons and positrons of the pair plasma itself, that acquire a drift velocity as they move along curved field lines and form two distinct populations of particles (Cheng \& Ruderman 1977), 3./ the interaction between electrons and positrons that form moving pair plasma clouds, that result from the "sparking' phenomenon arising above the surface of the star (Usov 1987).

These interactions were considered in the context of the Ruderman and Sutherland model, assuming weakly relativistic pair plasma and beam, in strict 
motion along magnetic field lines in the extremely strong pulsar field, and using a fluid description, equivalent to the choice of narrow peaked distribution functions of particles with a small spread in energy, $\Delta \gamma \ll \gamma$. In these conditions, the nonlinear stage of the second quoted process did provide correct agreement between derived and observed quantities such as frequencies, grow th rates, energy budget and luminosities (Asseo et al 1990; Asseo 1993; Asseo 1994).

Nevertheless, the analysis of the creation and repartition of pairs in standard models concludes to consequent large spreads in the energy of particles that form the pair plasma, $\Delta \gamma \approx \gamma$, that imply relativistic temperatures of particles. Thus, relativistic one-dimensional distribution functions are necessary to describe beam and pair plasma flows.

\section{Anisotropic relativistic pulsar pair plasma}

Pulsar pair plasmas are relativistic in two ways. First, because of the highly relativistic motion of the electrons and positrons forming the pair plasma. Second, because of the relativistic temperatures of pair plasma particles. In addition, they are anisotropic because of the extreme strength of the pulsar magnetic field.

The choice of distribution functions to characterize flows of relativistic particles in a pulsar magnetosphere is extremely important, as the dispersion relation for waves able to propagate depends on these very distribution functions. Using relativistic one-dimensional Juttner-Synge distribution functions, relativistic equivalent of Maxwellian distribution functions, we obtain analytical expressions of the dispersion relation for quasi-longitudinal waves, as a succession of terms whose coefficients $\zeta_{1}, \eta_{1}, \xi_{1}, \cdots$ depend on the temperature, velocities and Lorentz factors of the flows (Asseo \& Riazuelo, in these proceedings).

In the observer's frame, resonant 'Langmuir' waves from an ultra-relativistic beam and plasma interaction, have a high frequency $\omega \approx \sqrt{\gamma_{p}} \omega_{p} \mu^{-1 / 2}$, as $\mu \ll 3$. Hence, resonant unstable 'Langmuir' waves due to an interaction between the primary beam and secondary pair plasma in a pulsar magnetosphere, are probably not responsible for pulsar radio events, in analogy of Cheng \& Ruderman (1977) and Melrose et al (1999) conclusions. However, numerical analysis shows the possibility for an efficient two-stream instability to be raised up by the interaction between drifting electrons and positrons that form the ultra-relativistic pulsar pair plasma itself (Asseo \& Melikidze 1998).

\section{Pair plasma clouds moving in a pulsar magnetosphere}

Detailed analysis of the "sparking' phenomenon above the surface of a neutron star suggests that a succession of pair plasma clouds move along pulsar magnetic field lines. For such clouds, there are two different timescales on which a twostream instability develops : a long timescale $\approx 10 \mu$ s, related with stationary properties of pair plasma flows; a short timescale $\approx 1$ to 10 nanoseconds, associated with non-stationary 'sparking' phenomena. On one or the other timescale, dispersion relations that characterize wave propagation depend on the distribution functions of particles. To describe the assembly of pair plasma clouds and to introduce the stationary or the non-stationary character of particle flows in successively emitted clouds, we use appropriate arrangements of relativistic 
one-dimensional Gaussian distribution functions of the momentum modulated by secant hyperbolic functions that depend on the very localisation of the cloud (Asseo \& Melikidze 1998).

On the long timescale, our numerical analysis confirm the raising up of an effective two-stream instability associated with the interaction of electrons and positrons in relative drift motion within pair plasma clouds, assuming reasonable pulsar parameters together with a temperature parameter, $\mu \approx 1$. On the short timescale, overlapping and interaction of particles from successive pair plasma clouds, which have a large spectrum of energies, also lead to the starting of an efficient 'two stream'-like instability.

A comparison between the efficiency of two-stream instabilities on these two different timescales, shows that non-stationary effects may compete with or even dominate over drifting effects. In the limits of our linear and local treatment, this provides a new emission mechanism, eventually responsible for part of the high level of observed pulsar radio radiation (Asseo \& Melikidze 1998).

Besides, we know from the analysis of fluid equations that the two-stream instability nonlinearly evolves in the pulsar emission region towards a state of strong turbulence. More precisely, a lattice of regularly spaced Langmuir solitonlike structures probably exist, all with the same amplitude and characteristics (dimensions, timescales, charge, electric fields and energy content), that agree with observed microstructures (Asseo et al 1990; Asseo 1993; Asseo 1994).

J. Gil, G. Melikidze and A. Pataraya (these proceedings) propose a similar analysis for the curvature radiation from solitons, that involves additional contributions due to resonant particles at the group velocity of the waves, and use alike soliton characteristics. These contributions, modification of the coupling coefficient in the local-nonlinear term and nonlocal-nonlinear term arising from the nonlinear damping process, respectively introduce in the Nonlinear Schrödinger equation terms with coefficients $q$ and s. Specific values for $q$ and $s$, depend on distribution functions for pair plasma particles. For Gaussian distribution functions of the momentum considered by Melikidze et al in the observer's frame, the results are slightly modified as $q \geq s$. For a Maxwellian distribution function defined in the plasma rest frame, q can be kept unmodified while $\mathrm{s}$ is $k \lambda_{D}$ times smaller than q (Ichikawa et al 1973). This justifies our restriction to 'Langmuir' soliton-like solutions with zero velocity in the plasma rest frame, equivalent to the implicit assumption that the above contributions are negligible.

\section{Lattice of 'Langmuir soliton -like structures in the inhomogeneous weakly relativistic pulsar pair plasma}

In reality, the pair plasma flows along diverging magnetic field lines of a pulsar emission region and modified Langmuir waves and/or Langmuir solitons properties result from magnetic field and density gradients. Using a Lorentz transformation, but maintaining constant the value of the frequency as observed in the observer's frame, one shows that plasma wave propagation in an expanding strongly magnetized medium implies a wavelength decrease and an amplitude increase, as one moves away from the center of the star. Besides, resonant coupling between the interacting beam and plasma, and raising up of a two-stream instability, are necessarily strongly localized at the critical distance where $\omega_{0} \approx \omega_{p}(x)$. 
At some given distance, the two-stream instability is the source of 'Langmuir' soliton-like structures with both electrostatic and electromagnetic character, as $E_{\|}, E_{\perp}$ and $B_{\perp \perp}$ are coupled. ( $\|, \perp$ and $\perp \perp$ define directions relatively to the pulsar magnetic field). In the rest frame of the homogeneous pulsar pair plasma, $E_{\|}$is derived from a Nonlinear Schrödinger (NLS) equation, assuming a locally negligible $E_{\perp}$-contribution; $E_{\perp}$ is derived from a wave-like equation in which $E_{\|}$acts as a source; $B_{\perp \perp}$ is derived from Maxwell equations. In this way, 'the longitudinal part of the wave localizes the transverse wave': $E_{\|}$is a localized 'Langmuir' soliton-like component, while both $E_{\perp}$ and $B_{\perp \perp}$ are travelling 'localized' parts of the solution (Asseo et al 1990).

For the inhomogeneous pulsar pair plasma, the NLS equation includes a specific inhomogeneity term. 'Langmuir' soliton-like solutions of this equation exist and maintain their identity, the inhomogeneity only resulting in uniform acceleration of the solutions along the gradients. For instance with a linear density gradient, and assuming an initial soliton velocity $v_{0}=0$, the $\|$ component

$$
\begin{array}{r}
E_{\|}\left(x_{\|}, t\right)=a \lambda_{D} 2 \pi\left(\pi n_{0} k_{B} T\right)^{1 / 2} \operatorname{sech}\left[a\left(x_{\|}-x_{0}-v_{\|} t / 2\right)\right] \\
\exp i\left[x_{\|} v_{\|} / 2-v_{\|}^{2} t / 12\right]\left[\exp -i\left[\omega_{p 0}-\left(a^{2} \lambda_{D}^{2}\right) / 2\right] t+c . c .\right] .
\end{array}
$$

has stable structure but suffers uniform acceleration (Chen \& Liu 1978; Asseo et al 1990). Altogether, this allows description of a lattice of 'Langmuir' solitonlike structures in the pulsar emission region uniformly accelerated down the magnetic field and density gradients.

We conclude that in addition to radiation processes due to the electromagnetic components of 'Langmuir' soliton-like structures, to curvature radiation of these structures as they are dragged along magnetic field lines and to coherent acceleration of particles passing close, all studied in Asseo et al (1990) and Asseo $(1993 ; 1994)$, an additional radiation effect is to be associated with 'Langmuir' soliton-like structures in a pulsar emission region : it results from the acceleration of wave packets down the density gradient, gives rise to curvature radiation and provides further radio radiation in the pulsar magnetosphere.

\section{References}

Asseo E., Pelletier G., Sol H. 1990, MNRAS 247, 529

Asseo E. 1993, MNRAS 264, 940

Asseo E. 1994, Physica Scripta 52, 87

Asseo E., Melikidze, G.I. 1998, MNRAS 301, 59

Asseo E., Riazuelo, A. submitted to MNRAS (1999)

Chen H.H, Liu C.S. 1978, Phys. Fluids 21, 377

Cheng A.F., Ruderman M.A. 1977, ApJ 212, 800

Ichikawa Y., H. Suzuki, T. Taniuti, T. 1973, J. Phys.Soc. Japan, 34, 1089

Melrose D.B., Gedalin, M.E., ApJ submitted (1999) (preprint)

Ruderman M.A., Sutherland P.G. 1975, ApJ 196, 51

Usov V.V., 1987, Ap.J., 320, 333 